\title{
Coupling of Dispersive Tsunami Propagation and Shallow Water Coastal Response
}

\author{
F. Løvholt*, G. Pedersen and S. Glimsdal
}

NGI, PO Box 3930 Ullevål Stadion, NO-0806 Oslo, Norway

\begin{abstract}
The key issue of this article is the concept of combining a model dedicated to dispersive large scale propagation of tsunamis with ComMIT, developed and made freely available by NOAA, that is a state of the art tool for tsunami impact studies. First, the main motivation for this approach, namely the need for efficient computation of runup of tsunamis from submarine/subaerial slides and certain types of earthquake, is discussed. Then the models involved are presented. We describe in some detail the dispersive model component which is a Boussinesq type model that is recently developed for tsunami propagation purposes. Finally, the performance and flexibility of the joint model approach is illustrated by two case studies including inundation computations at selected cites. The potentially disastrous, but small probability, flank-collapse event at the La Palma Island is used as an example of slide generated tsunamis where dispersion plays an important role. The second example is a tsunami from a potential inverse thrust fault at the Lesser Antilles. In this case dispersion during propagation is important for some regions, but not for others.
\end{abstract}

Keywords: Tsunamis, dispersive water waves, model coupling, run-up.

\section{MOTIVATION}

Tsunamis are gravity waves set in motion by large sudden changes of the water, having characteristics intermediate between tides and swells. The far-field tsunami propagation is usually modelled with some set of depth integrated, long wave models. The simplest and most used models for the propagation phase are the shallow water equation sets denoted as LSW (linear) and NLSW (nonlinear). These equations are simple, well suited for explicit time stepping and special phenomena like breaking bores and inundation may be included. NLSW type models are by far the most popular in tsunami run-up modelling. A number of operational NLSW for use in tsunami run-up calculations are currently available, for instance ComMIT/MOST $([1,2,3])$, COMCOT [4], TUNAMI-N2 [5], and GEOCLAW [6]. These NLSW type models exhibit features like breaking/bore capturing, inundation of dry land, friction terms, and nesting capabilities or adaptive grids, among others. The above mentioned functionality of the operational NLSW type models make them popular for modelling inundation in real case tsunami applications.

For most aspects of tsunami modeling shallow water theory is probably the best option in view of efficiency, software implementation and performance combined. However a dispersive wave model may sometimes be needed. Tsunamis generated by non-seismic sources are often too short to be adequately described by shallow water theory. A global propagation analysis may require dispersive models even for earthquake generated tsunamis. In addition

*Address correspondence to this author at the NGI, PO Box 3930 Ullevål Stadion, NO-0806 Oslo, Norway; Tel: (+47) 220230 43;

Fax: (+47) 222304 48; E-mail: finn.lovholt@ngi.no there are particular phenomena, such as undular bores, that are genuinly non-hydrostatic and will be lost in the shallow water simulations. Dispersive waves are most generally described by the primitive wave equations (Euler or NavierStokes). However, in most tsunami applications wavelengths of more than a few water depths are dominating, which suggest the use of Boussinesq type equations, with standard or optimized dispersion properties. To be suited for tsunami simulations a Boussinesq model should be efficient enough to allow trans-oceanic computations, on desktops preferably. Geographical coordinates and the Coriolis effect must be included. Optimized dispersion properties may sometimes be advantageous, while higher order nonlinear terms rarely are of interest during propagation. We have recently developed a Boussinesq model (GloBouss) that is primarily intended for long distance tsunami propagation.

Herein, we focus on a combined model approach where GloBouss is combined with ComMIT/MOST that is an operational shallow water tsunami model suitable for compuation of coastal impact and runup. We argue that the inundation part of the MOST model is robust, effcient, flexible and well tested, while existing Boussinesq models may miss a little on run-up/drawdown, wave breaking and computer efficiency [7]. The significance of dispersion depends on the ratio $(h / \lambda)^{2}$, where $h$ is the depth and $\lambda$ is a measure of the wavelength. For many slide generated tsunamis, as well as a few seismic ones, this ratio may be of order $1 / 100$, or larger, in the deep ocean. Hence, dispersive effects may be important, depending on the propagation distance. According to geometrical optics for normally incident waves the ratio $(h / \lambda)^{2}$ will be reduced in proportion to $h$ during shoaling, which means that the wave motion in coastal regions is hydrostatic. However, 
exceptions may occur due to the nonlinear steepening of the wave fronts. Under certain conditions dispersion may be crucial for the dynamics of the short fronts and undular bores (fission of the wave-front prior to breaking) may evolve. We realize that the NLSW models may not include such effects. Still, as the undular bores are short in length, and should dissipate more rapidly than the low frequency part of the tsunami, a proper handling of features like bore capturing and dry land inundation during breaking will often be more important for the inundation of land.

In addition, the model coupling provides also the possibility of including sources independent of the ComMIT interface. This is relevant for some types of seismic tsunami sources, but perhaps more importantly, allows the inclusion non-seismic sources such as landslides.

Many LSW models inherit numerical dispersion of a form similar to the real dispersion in a standard Boussinesq model. An attractive idea is then to adjust the model parameters in such a way that the numerical dispersion mimics the physical one. For the MOST method this is demonstrated in [8], while corresponding features are discussed [9] for the LSW counterpart to the GloBouss model. However, there are serious problems related to turning this idea into a useful computational strategy. For traditional LSW models reproduction of physical dispersion requires a specific relation between depth and grid increments to be fulfilled. In one horizontal direction (1HD) this is rather straightforward, even though the grid must be coarse, but it is hardly achievable for structured grids in 2HD. Moreover, the anisotropy of the numeric dispersion will then anyhow prevent an accurate reproduction of dispersion. In special methods [10,11] some of the difficulties are eliminated, but problems linked to coarse grids and stability remain. Firstly, moderately short waves for which an improved Boussinesq model, such as that used herein, is still good can hardly be resolved at all. Secondly, the grids are too coarse to allow a good representation of bathymetric effects and nonlinearity. Subsequently we will demonstrate that even oceanic propagation of dispersive waves can be quite efficiently modelled using physically well-founded Boussinesq equations. At present we believe this to be the sound approach.

This paper is organized as follows. First, we present the formulation of the global Boussinesq solver (GloBouss), and review some key elements of the numerical implementation. Next, the model coupling is presented. Finally, we give some examples of results from numerical simulations of a potential a seismic scenario towards Bridgetown, Barbados, as well as the hypothetical La Palma tsunami inundating the city of Cadiz in Spain.

\section{MODELS}

\subsection{The ComMIT Model}

The abbreaviation MOST (Method of Splitting Tsunamis) accounts for the numerical wave model, whereas ComMIT is the user interface. The runup model is based on the depth integrated non-linear shallow water equations (NLSW), and is based on a characteristic formulation solved with a Godunov type scheme for shock capturing, combined with operator splitting. Moreover, ComMIT offers the possibility of including three levels of nested grids labelled $\mathrm{A}, \mathrm{B}$, and $\mathrm{C}$ respectively, with the $\mathrm{C}$-grid having the finest resolution. This enables computation of the runup in relatively fine resolutions, a feature that is now standard in most operational standard inundation models of the NLSW type.

The runup model is also extensively verified, for instance towards experimental data on runup of solitary waves in 1HD [2], and in 2HD towards field observations due to the runup of the 1993 Okushiri tsunami [3]. The latter case has inspired a set of laboratory experiments that serve as a benchmark problem for testing operational tsunami models with runup [12].

\subsection{Brief Review of Dispersive Long Wave Models}

The Boussinesq equations come in great diversity; the standard formulation [13,14], Serre's equation, Green-Nadgi and many more. Since the mid nineties Boussinesq equations with improved dispersion properties [15] and full nonlinearity [16-19] have been incorporated in general purpose models. Excellent examples are the FUNWAVE [20-22] and COULWAVE [23] models that are freely available and widely used. These models are useful also for tsunami modeling, but they may be somewhat too computationally demanding for large scale computation. In addition their strength, the full non-linearity, is mainly useful in very shallow water. Here the waves rapidly break and it is doubtful that the Boussinesq models then are as good, or robust at least, as NLSW models with bore capturing facilities [18]. In addition the available versions of these models do not include geographical co-ordinates or the Coriolis effect, even though the implementation of such effects in FUNWAVE has been briefly reported [24]. The new propagation model presented herein is not an general alternative to models such as FUNWAVE/COULWAVE, but a simpler option with emphasis on efficiency and only standard nonlinear terms.

\subsection{Boussinesq Equations}

To write the equations in standard form we introduce dimensionless variables according to

$$
\begin{array}{ll}
(\psi, \phi)=\Theta(\hat{x}, \hat{y}), \quad \hat{t}=\frac{R \Theta}{\sqrt{g h_{0}}} \\
(u, v)=\varepsilon \sqrt{g h_{0}}(\hat{u}, \hat{v}) \quad h=h_{0} \hat{h} \quad \eta=\varepsilon h_{0} \hat{\eta}
\end{array}
$$

where $\psi$ and $\phi$ are the longitude and latitude respectively, the hats indicate dimensionless variables, $g$ is the constant of gravity, $h_{0}$ is a characteristic depth, and $\varepsilon$ is an amplitude factor. The characteristic horizontal length (wave length) now becomes $L_{C}=R \Theta$, which may determine $\Theta$, and the "long wave parameter" is accordingly recognized as $\mu^{2}=h_{0}^{2} /\left(R^{2} \Theta^{2}\right)$. For the physical constants we substitute for $g=9.81 \mathrm{~m} / \mathrm{s}^{2}$ and for the Earth's equatorial radius 
$R=6378135 \mathrm{~m}$. It is recognized that these quantities are not constant, but their variation is neglected along with other small effects of the rotation and curvature of the Earth.

Rotational effects are included simply by adding the Coriolis term to the momentum equation. According to the length and time scale inherent in (1) we obtain a nondimensional Coriolis parameter $f=2 \Omega R \Theta \sin \phi / \sqrt{g h_{0}}$, where $\Omega$ is the angular frequency of the Earth.

By omission of the hats the dimensionless equation of continuity in geographical coordinates reads

$c_{\phi} \frac{\partial \eta}{\partial t}=-\frac{\partial}{\partial x}[(h+\varepsilon \eta) u]-\frac{\partial}{\partial y}\left[c_{\phi}(h+\varepsilon \eta) v\right]-c_{\phi} \frac{\partial h}{\partial t}$

where $c_{\phi}=\cos \phi$ and $u, v$ are interpreted as vertically averaged velocity components. The momentum equations read

$$
\begin{aligned}
& \frac{\partial u}{\partial t}+\varepsilon\left(\frac{u}{c_{\phi}} \frac{\partial u}{\partial x}+v \frac{\partial u}{\partial y}\right)=-\frac{1}{c_{\phi}} \frac{\partial \eta}{\partial x}+f v-\gamma \mu^{2} h^{2} \frac{1}{c_{\phi}} \frac{\partial D_{\eta}}{\partial x}+\mu^{2} S_{x} \\
& +\frac{\mu^{2}}{2} \frac{h}{c_{\phi}^{2}} \frac{\partial}{\partial x}\left[\frac{\partial}{\partial x}\left(h \frac{\partial u}{\partial t}\right)+\frac{\partial}{\partial y}\left(c_{\phi} h \frac{\partial v}{\partial t}\right)\right] \\
& -\mu^{2}\left(\frac{1}{6}+\gamma\right) \frac{h^{2}}{c_{\phi}^{2}} \frac{\partial}{\partial x}\left[\frac{\partial}{\partial x}\left(\frac{\partial u}{\partial t}\right)+\frac{\partial}{\partial y}\left(c_{\phi} \frac{\partial v}{\partial t}\right)\right]
\end{aligned}
$$

$\frac{\partial v}{\partial t}+\varepsilon\left(\frac{u}{c_{\phi}} \frac{\partial v}{\partial x}+v \frac{\partial v}{\partial y}\right)=-\frac{\partial \eta}{\partial y}-f u-\gamma \mu^{2} h^{2} \frac{\partial D_{\eta}}{\partial y}+\mu^{2} S_{y}$

$+\frac{\mu^{2}}{2} h \frac{\partial}{\partial y}\left[\frac{1}{c_{\phi}} \frac{\partial}{\partial x}\left(h \frac{\partial u}{\partial t}\right)+\frac{1}{c_{\phi}} \frac{\partial}{\partial y}\left(c_{\phi} h \frac{\partial v}{\partial t}\right)\right]$

$-\mu^{2}\left(\frac{1}{6}+\gamma\right) h^{2} \frac{\partial}{\partial y}\left[\frac{1}{c_{\phi}} \frac{\partial}{\partial x}\left(\frac{\partial u}{\partial t}\right)+\frac{1}{c_{\phi}} \frac{\partial}{\partial y}\left(c_{\phi} \frac{\partial v}{\partial t}\right)\right]$,

where $D_{\eta}$ is the dimensionless Laplacian of $\eta$

$D_{\eta}=\frac{1}{c_{\phi}}\left[\frac{\partial}{\partial x}\left(\frac{1}{c_{\phi}} \frac{\partial \eta}{\partial x}\right)+\frac{\partial}{\partial y}\left(c_{\phi} \frac{\partial \eta}{\partial y}\right)\right]$.

Terms due to the time dependent depth do appear both in the continuity equation and as the higher order terms in the momentum equations. The principal contribution is the source term in the continuity equations, while the less important terms $\mu^{2} S_{x}$ and $\mu^{2} S_{y}$ are neglected in the momentum equation. A description of the omitted terms, together with a test of their influence on a benchmark problem, is found in [25]. See also [26].

Putting $\gamma$ equal to zero we retrieve the standard Boussinesq equations [14], while the $\gamma=-0.057$ yields the same improved dispersion properties as in the formulation of [15].

\subsection{The GloBouss Model}

The finite difference method for solving the set (2) and (3) is developed from the model employed and documented in [27] and [28]. Like a number of shallow water models, as well as a few Boussinesq models, such as [22] and [29], we employ the staggered C-grid [30] in the spatial discretization. Unlike [22, 29], but similar to some hydrostatic models, we employ a staggered grid also in time with nodes for surface elevation and velocities alternating along the time axis. We will not spell out the discrete equations herein, but refer to the report [9] for details. Instead we will summarize the model properties.

Applying a staggered grid in space and time, we replace all the linear derivatives in (2) and (3) by symmetric, centered differences. This yields a more accurate temporal resolution than [29] and a much simpler time stepping procedure than the multi-level predictor/corrector method employed in the FUNWAVE [20-22] and COULWAVE [23] models. An example of numerical dispersion relation for the optimized $(\gamma=-0.057)$ version of GloBouss is shown in

Fig. (1), also compared with the analytical dispersion relations for the Boussinesq models as well as full potential theory. Hence, for certain (fine) grid resolutions, the numerical dispersion relation is in better agreement with the full potential theory than the corresponding analytical relation for the short wave components. Unlike the analytical Boussinesq equation, the discrete model yields the correct limit $c=0$. In the non-linear terms, the Coriolis term and coefficients we also employ symmetric averaging.

Numerical correction terms are included to obtain a fourth order method for the dominant LSW balance $(\mu, \varepsilon \rightarrow 0)$ of the equations. In some respects these resemble the higher order spatial differences in FUNWAVE, but for the present model we must include temporal corrections as well. However, due to the staggered grid and the one-level temporal scheme the corrections must be transformed by means of the leading order balance of the Boussinesq equations. This results in additional discrete terms akin to the dispersion terms normally appearing in Boussinesq type equations. In a forerunner model $[27,28]$ a similar procedure was applied to obtain an improved numerical dispersion relation, but not a full fourth order scheme for the LSW part of the Boussinesq equations. The related model [29] did not include higher order numerical representations.

Land is represented as staircase, no-flux boundaries. Such boundaries have been shown to function well, even in the nonlinear case, when situated in water of sufficient depth [9]. Moreover, with the computational stencil (Fig. 2), fictitious boundary cells are avoided. On the other hand, dry cells during withdrawal cause problems and may not be permitted. At open boundaries we employ sponge layers. Runup facilities is included by a one-way nesting with ComMIT runup model [1-3], see below for details.

When non-linearity, the Coriolis force or dispersion are retained, both the continuity and momentum equations yield implicit sets of equations to be solved at every time steps. The temporally staggered grid allows the implicit continuity 


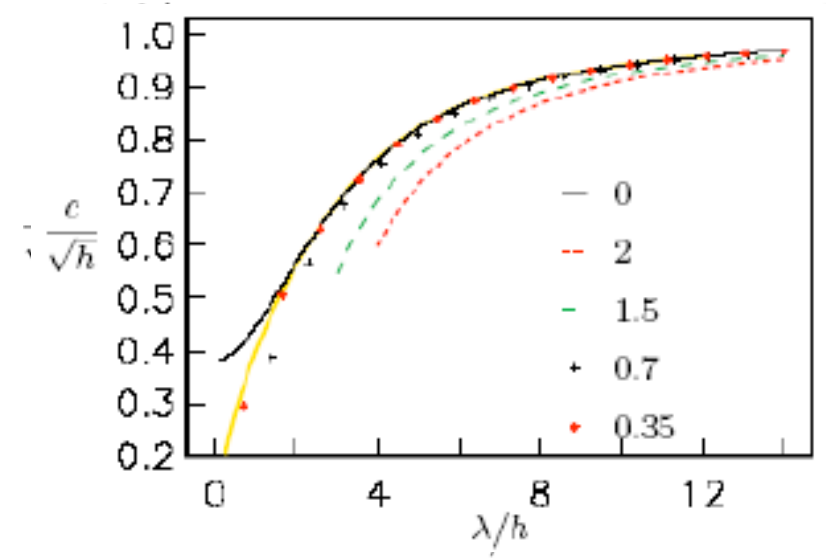

Fig. (1). Phase velocities for the Boussinesq equations with $\gamma=-0.057$ and direction of wave advance $\theta=0^{\circ}$. The bold yellow line depicts the phase velocity according to full potential theory and the solid black line the analytical phase velocity for the Boussinesq equations. The dashed and dotted curves show the phase velocities for the discrete equations. The legend indicate the value of $c_{\phi} \Delta x / h$. The other grid increments in the discrete case are $\Delta y=c_{\phi} \Delta x / h$ and $\Delta t=1 / 2 \sqrt{h} c_{\phi} \Delta x / h$

equation set to be decoupled from momentum equation sets. The equation sets are solved by an ADI (alternating direction implicit) iteration iteration scheme. For the present form of the Boussinesq equations this implies alternating implicit sweeps in the $x$ and $y$ components of the momentum equation, similar to the approach of [29]. Two sweeps do suffice for the applications herein, but for cases dominated by shorter waves, the number of iterations would have to be increased. Yet, in other cases a single sweep may suffice. The iteration scheme is somewhat simpler than that of $[27,28]$ and much simpler, as well as more efficient, than the one used in the FUNWAVE and COULWAVE models.

Instabilities due to steep depth gradient may occur for certain depth configurations. The occurrence of instabilities due to steep gradients are indeed common in a variety of Boussinesq models in linear form [31]. In [9], it is shown that the stability limits in the optimized version of the model $(\gamma=-0.057)$ arises for extreme depth changes ( $\Delta h$ close to unity and small $\Delta x$ ) only.

\subsection{Source Implementation in GloBouss}

For the simulation of tsunamis from earthquakes the vertical uplift of the seabed is generally copied onto the surface as an initial condition, sometimes slightly modified to avoid sharp and unphysical gradients [32]. GloBouss can read initial conditions for the surface elevation and the velocity components. The latter is primarily useful for testing purposes and for importing data from refined simulations for smaller regions, that may include the source of the waves, or from other tsunami models.

Submarine slides, on the other hand, are much slower processes than earthquakes, and the wave generation models thus require a source representation of finite extent in time and space. One of the simplest options is to specify a pre- defined sink/source distribution in a long wave model. This enables an approximate description of mass gravity flows that are parameterized or described by separate models.

The source distribution, $q(x, y, t) \equiv-\partial h / \partial t, \quad$ is represented by a sequence of spatial fields $q^{(n)}$, for $n=1, \ldots, N_{t}$. Correspondingly, a set of times $T_{0}, \ldots, T_{N_{t}}$ is defined and $q^{(n)}$ is interpreted as the source distribution acting in the time interval $\left[T_{n-1}, T_{n}\right]$. The temporal resolution may be nonuniform, but the fields must be numbered chronologically. Generally, the times $T_{n}$ will not coincide with a temporal grid point. The effective sources invoked in the equation of continuity may then be composed of sums of subsequent $q^{(n)}$ including fractional contributions. Eventually, the total added volume becomes a discrete, spatial integral of $\sum_{p=1}^{N_{t}}\left(T_{p}-T_{p-1}\right) q^{(p)}$.

Each field, $q^{(n)}$, must be specified on a rectangular grid, $G^{(n)}$, that may be different for each $n$, but must comply to the same coordinate system as the depth matrix. The source field is then extended to the whole $x, y$ plane by setting $q^{(n)}$ to zero outside $G^{(n)}$ and by interpolation withing $G^{(n)}$. The interpolation is based on either bi-linear polynomials, which is generally recommended, or bi-quadratic splines, that should be used only with great caution. The depth may also be modified due to the accumulated source distribution.

\subsection{Verification and Testing}

In Fig. (3) the performance of GloBouss for solitary wave propagation is studied. The deviation from the exact solution is small. More significantly, the amplitudes in the simulations approach constant values in time. This corresponds to the existence of a slighty modified numerical solitary wave, without inherent damping [33]. Such a property depends crucially on the discretization and may not be obtained when asymmetric differences are used. GloBouss in its present form is particularly adapted to long distance propagation of linear or non-breaking waves. If breaking is to be included, other discretizations, in particular for the nonlinear terms, may be more favorable.

The coding of GloBouss has been verified by comparison with preceding models for cases where the solutions should be identical, save for effects of finite arithmetics. Typically the relative errors are less than $10^{-6}$, allowing for some error accumulation, for the 32 bits version of the codes.

For test purposes we have studied the linear shoaling properties for waves incident to a simple square-cosine shaped shelf, consisting of two uniform depth regions, with non-dimensional depths 1 and $h_{r}$, respectively, joined by a smooth slope (see first panel of Fig. 4).

Different Boussinesq formulations, including the present, the standard one and that of Nwogu [15], are compared to 


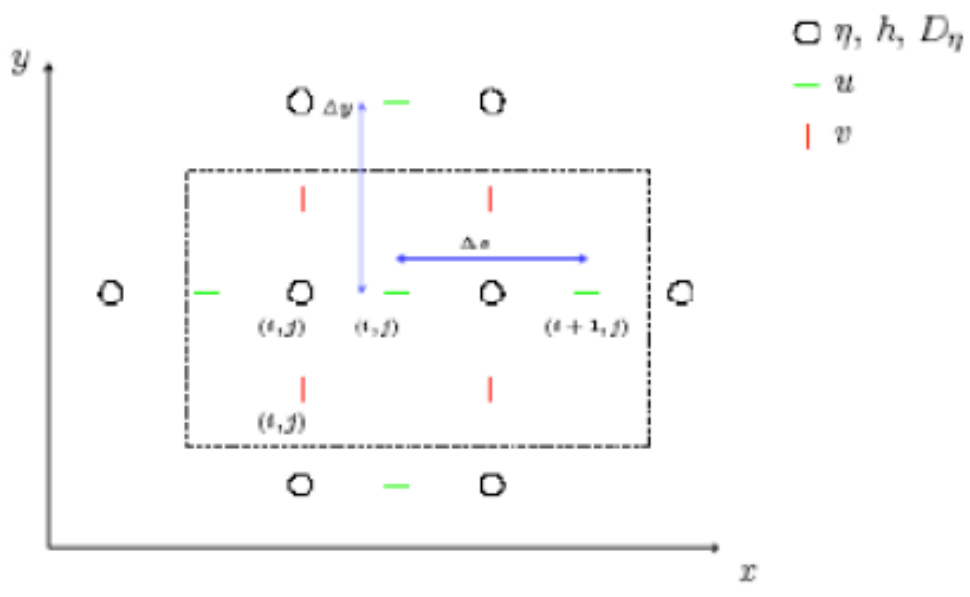

Fig. (2). Computational stencil, centered at point $(i, j)$, for the $x$-component of the momentum equation. In the linear case without corrected dispersion only the nodes within the dashed box are involved. The $u$ and $\eta$ nodes outside the box enter through the non-linear term and $D_{\eta}$, respectively.

full potential theory by evaluation of the $L_{2}$ norms of the deviation. For reference also the $L_{2}$ norms of deviations due to grid refinement have been computed. For the grid increments employed, these are smaller than the differences between the models. The present model with $\gamma=-0.057$ and Nwogu's with $z_{\alpha}=-0.531 h$ are equivalent in constant depth. However, the two models are not identical for variable depth. Besides, a small discrepancy is also introduced through the treatment of the initial conditions.

A test case with a short initial wave elevation $(\lambda=3)$ and a bottom gradient $(\ell=2)$ is depicted in Fig. (4). From the right panels we observe that the pre-existing and current standard formulations are virtually identical, while the performances of the current optimized model and the Nwogu model are similar. The optimized formulations are superior to the standard formulations for this case, in particular near the first trough, but they display a marked deviation from full potential theory at the front due to the large depth gradient. In the left panels the solution, at different times, and the accuracy of the potential model are shown.

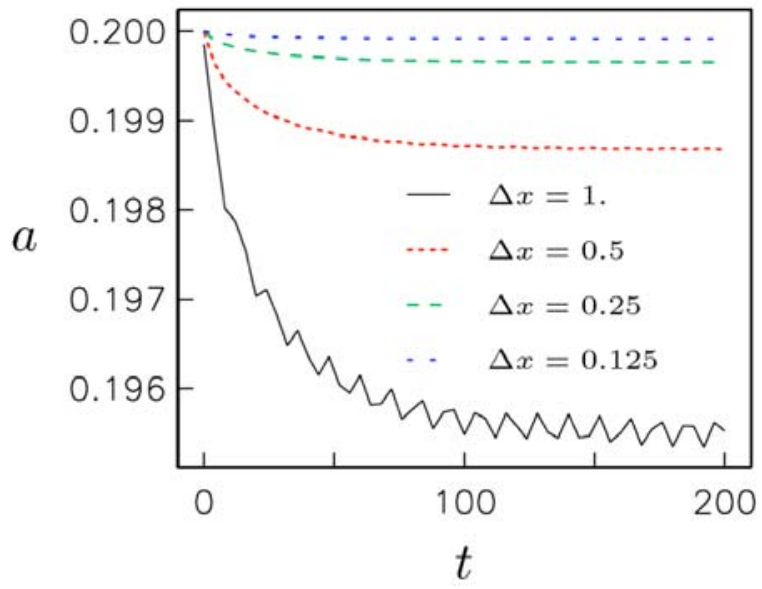

Other tests include investigations of eigenoscillations in basins and diffraction of solitary waves by a vertical cylinder. Details of the model validation tests are given in [9].

\subsection{Coupling with ComMIT}

By using a one way nesting procedure, ComMIT reads the output from the propagation model over the model boundaries at each time step. As the propagation and runup models are generally operating on different grids, the boundary values obtained from propagation model are interpolated to the runup model by bi-linear interpolation in space and linear interpolation in time. Technically, the nesting is performed by dumping so-called propagation files from GloBouss, containing time dependent fields of the surface elevations and the two velocity components over a region covering the whole computational domain of the local model. The file format of the propagation files are of the NetCDF type. From GloBouss, NetCDF propagation files compatible with the ComMIT input are produced over a user defined region and with a user defined resolution.

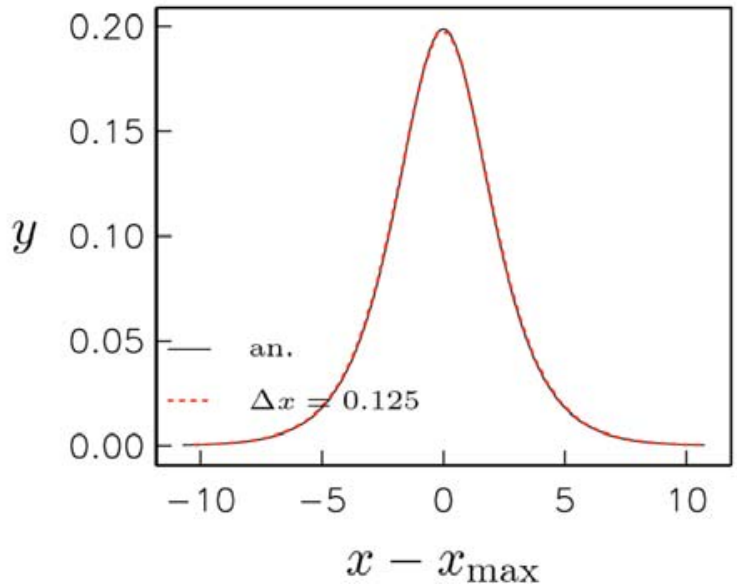

Fig. (3). Left: time evolution of the amplitude $a(t)$ of undular bores for different grid resolutions $\Delta x$ (indicated by the legend). Right: comparison with of a numerical simulation with an analytical solitary wave shape, with amplitude $A=a(t)$, after $t=200$ for $\Delta x=0.125$. 

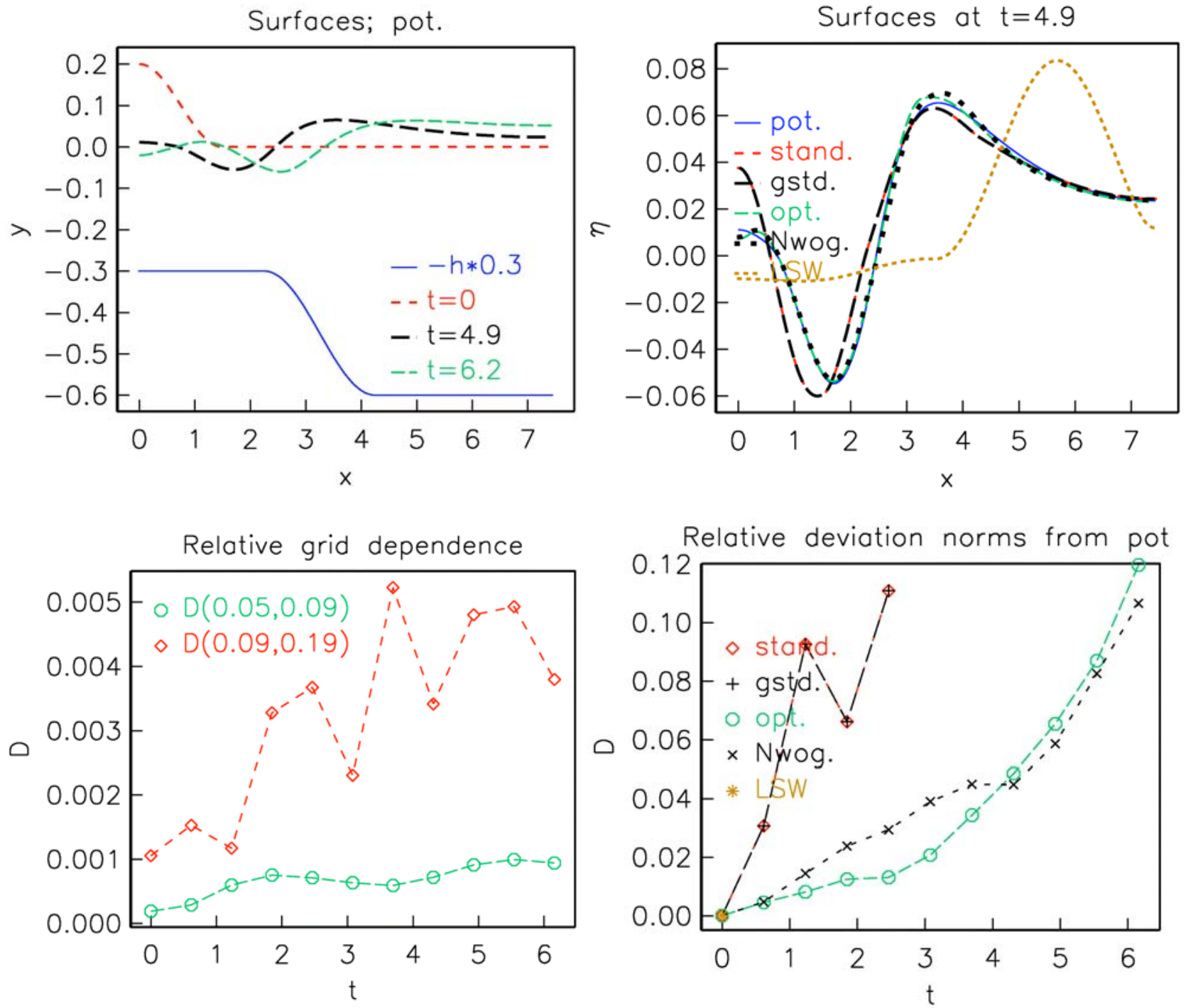

Fig. (4). Comparison of linear Boussinesq and full potential models. Upper left panel: surfaces at given times and the bottom. Upper right panel: comparison of different solvers at $t=4.9$. Lower left panel convergence of potential model; $D\left(\Delta x_{1}, \Delta x_{2}\right)$ is the $L_{2}$ norm of the difference between the solutions with grid increment $\Delta x_{1}$ and $\Delta x_{2}$, respectively. Lower right panel: difference between other model sand the potential theory. The LSW solution rapidly grows beyond the depicted window. Explanation of legends: stand.: pre-existing standard formulation. gstd.: current standard formulation .opt.: current optimized formulation $(\gamma=-0.057)$. Nwog.: Nwogus formulation. LSW: shallow water equations.

\section{EXAMPLE STUDIES}

\subsection{Runup in Cadiz from a Hypothetical Landslide Generated Tsunami off La Palma Island}

Potential large-scale landslides emerging off the La Palma Island, represents a potential but unlikely, regional tsunami threat to coastlines in the Atlantic Ocean [34]. After the work of [35] resulting in large public attention, landslide generated waves from La Palma has been studied in a series of papers [36-38]. [38] illustrated that unlike tsunamis of seismic origin, the worst case La Palma scenario could generate a dispersive wave-train where amplitudes in the trailing waves exceeded the leading wave. Thus, a dispersive model is needed.

Here, we study the inundation in the city of Cadiz due to the La Palma landslide scenario of [38], having a volume of
$375 \mathrm{~km}^{3}$ and a maximum velocity of $150 \mathrm{~m} / \mathrm{s}$ [37]. A snapshot of the wave propagation modelled with GloBouss after 1 hours 45 minutes towards Morocco, Spain, and Portugal is shown in Fig. (5), displaying multi-crested dispersive characteristics. For a grid covering $90^{\circ} \times 70^{\circ}$ (see Table 1) an simulation for 8 hours propagation time (2264 time steps) was computed in 3 hours 50 min using a single thread on an Intel(R), Quad CPU, Q6700, 2.66 Ghz computer with $8 \mathrm{~Gb}$ RAM. Using grid refinement tests, accuracy of $1-4 \%$ on a 2 ' grid was obtained for the wave propagation near the Canaries, with better accuracy for the leading wave than for the trailing waves [38]. The surface elevation displayed in Fig. (5) as well as the corresponding velocity fields were used as initial condition in the subsequent simulation coupled with runup in Cadiz. For the runup computations, three levels of nested grids were applied, their resolutions and number of grid points are listed 
in Table 1. On a laptop with $2.4 \mathrm{GHz}$ Intel Core 2 Duo processor with $3 \mathrm{~GB}$ RAM, the CPU time was 21 minutes for 3 hours and 8 minutes of real time propagation. Fig. (6) show snapshots of the inundation displayed for the finest grid with a grid resolution of $130 \mathrm{~m}$. As the waves become shorter and steeper, they break before inundating the coastline (Fig. 6), giving runup of up to $20 \mathrm{~m}$. The trailing waves are shorter and higher than the leading one, and in addition, they meet the ocean ward current resulting from the leading trough. Hence, they break further offshore. It is noted that undular bores evolved in the front of the tsunami impacting North America [38]. It is not unlikely that undular bores could evolve in a similar fashion towards Cadiz.

Table 1. Number of Grid Cells and Grid Resolution for the Cadiz and Bridgetown Test Cases

\begin{tabular}{|c|c|c|cc|}
\hline Case & Grid & $\begin{array}{c}\text { Number of } \\
\text { Points }\end{array}$ & Resolution \\
\hline \hline Cadiz & GloBouss & $2700 \times 2100$ & $2^{\prime}$ & $3.7 \mathrm{~km}$ \\
\hline Cadiz & ComMIT-A & $300 \times 294$ & $1^{\prime}$ & $1.85 \mathrm{~km}$ \\
\hline Cadiz & ComMIT-B & $372 \times 360$ & $1 / 4^{\prime}$ & $0.47 \mathrm{~km}$ \\
\hline Cadiz & ComMIT-C & $420 \times 420$ & $1 / 16^{\prime}$ & $0.09 \mathrm{~km}$ \\
\hline Bridgetown & GloBouss & $1081 \times 721$ & $1^{\prime}$ & $1.85 \mathrm{~km}$ \\
\hline Bridgetown & ComMIT-A & $25 \times 30$ & $1 / 8^{\prime}$ & $0.25 \mathrm{~km}$ \\
\hline Bridgetown & ComMIT-B & $49 \times 66$ & $1 / 16^{\prime}$ & $0.1 \mathrm{~km}$ \\
\hline Bridgetown & ComMIT-C & $268 \times 334$ & $1 / 120^{\prime}$ & $0.015 \mathrm{~km}$ \\
\hline
\end{tabular}

\subsection{Runup Studies in Bridgetown, Barbados}

The Norwegian Geothecnical Institute (NGI) and the University of the West Indies (UWI) have participated in a capacity building program on natural disaster mitigation in the Caribbean. One of the deliverables was a Disaster Mitigation Demonstration Project (DMDP) focusing on an example tsunami risk assessment for the city of Bridgetown, Barbados, see [39]. In this section we present results from the study related to the tsunami inundation modelling.

We employ results from a numerical simulation of a potential tsunami earthquake scenario at the Lesser Antilles (east of Guadeloupe, see Fig. 7) obtained by the GloBouss model. The Lesser Antilles scenario has a dip angle of $80^{\circ}$, mean slip 4m, width $55 \mathrm{~km}$, total length $191 \mathrm{~km}$. For a shear strength of $30 \mathrm{MPa}$ this gives a moment magnitude $M_{w}=8.0$, which is considered a 'credible worst case scenario' with a return period in the order of several hundred years. As for the Cadiz example, we apply three levels of grids, as listed in Table 1. On a $2.4 \mathrm{GHz}$ Intel Core 2 Duo processor with $3 \mathrm{~GB}$ RAM, the CPU time was 44 min $40 \mathrm{~s}$ for 3 hours of real time propagation.

Fig. (7) shows a snapshot of the simulated surface elevation after 30 minutes of propagation. The maximum surface elevations imply that the Lesser Antilles scenario affects mostly the eastern part of the Lesser Antilles. The highest waves are propagating in the east-west directions. The sea surface elevation around the generation area is 1-4 $\mathrm{m}$, while the islands south and north of the most affected ones have sea surface elevations off the shore of above 0.5 $\mathrm{m}$. There is some effect of the tsunami found also in the southern part of Puerto Rico and along the north-western

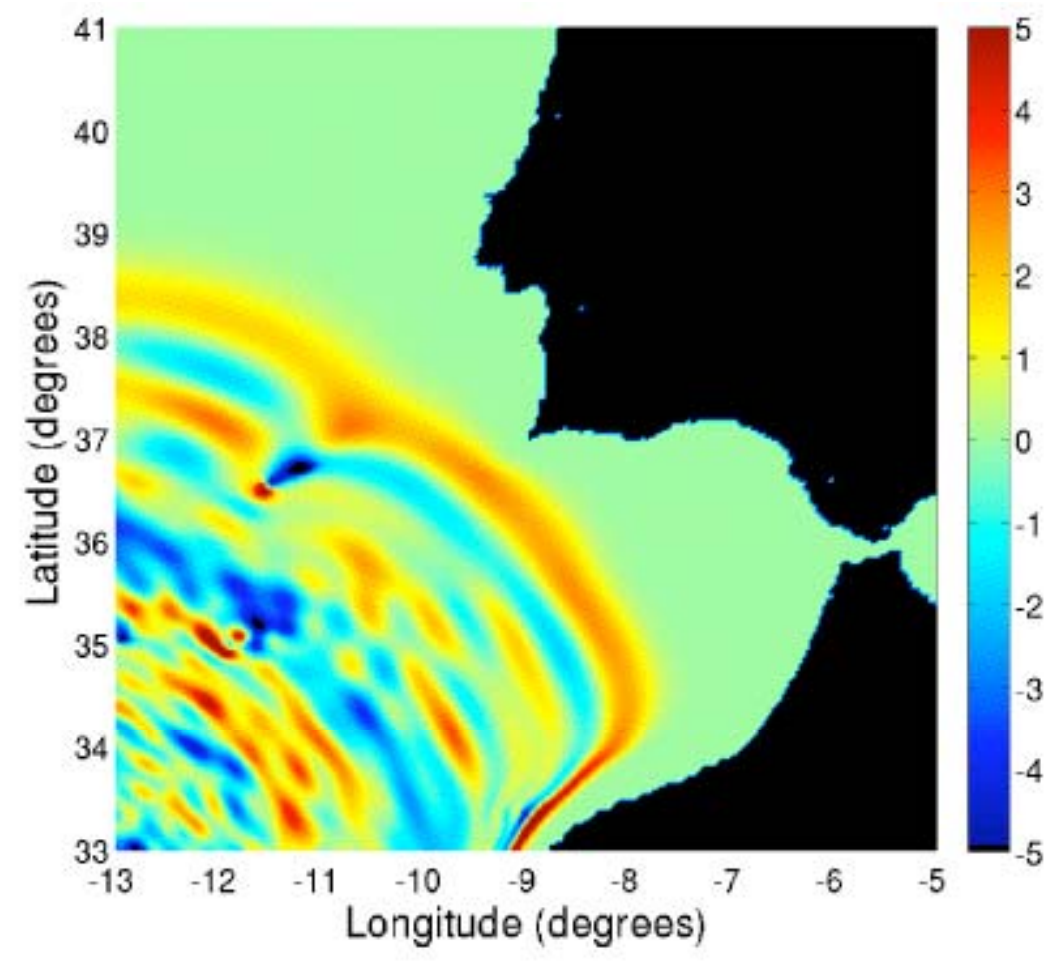

Fig. (5). Surface elevation from the worst case La Palma Island scenario after 1 hour and 45 minutes of propagation. 


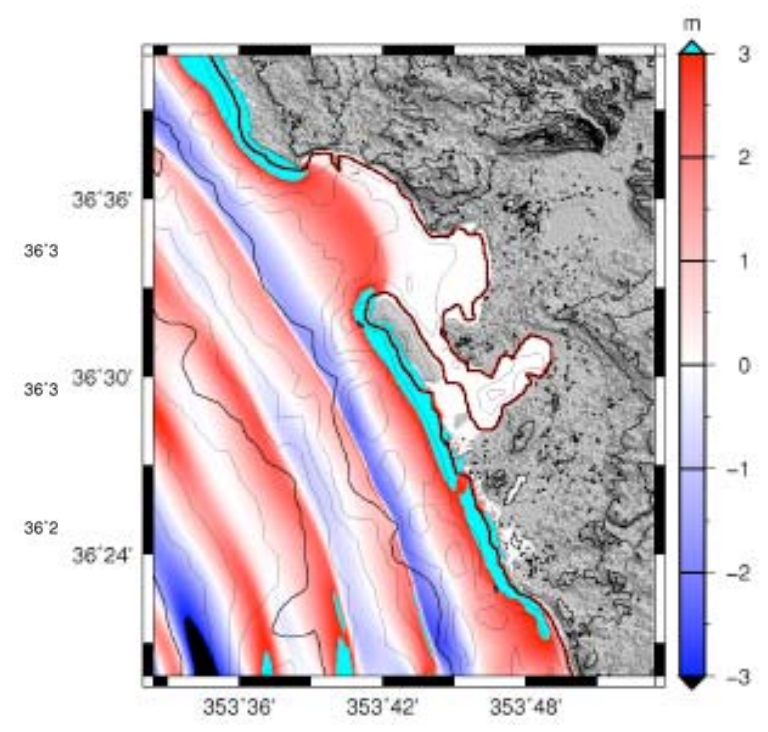

(b)

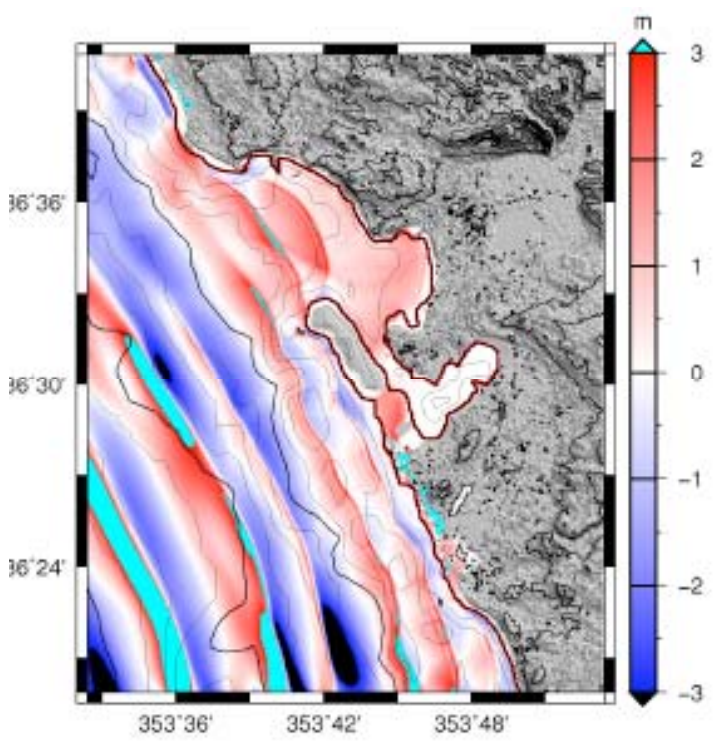

Fig. (6). Inundation from the La Palma scenario towards Cadiz. Upper panel, snapshot of the inundation after 2 hours and $39 \mathrm{~min}$. Lower panel, snapshot of the inundation after 2 hours and $50 \mathrm{~min}$.

coast of Venezuela. The rest of the Caribbean Sea is only slightly affected. It is clear that the area west of the Lesser Antilles/West Indies is protected by the islands closest to the source area.

In the propagation simulation we employ a $1^{\prime}$ GEBCO grid. Fig. (8) displays the effect of the resolution on times series extracted at $200 \mathrm{~m}$ depth outside Bridgetown, Barbados. This depth is roughly representative for the limits of the A grid (largest grid) used in ComMIT. The leading through and crest is reproduced very well for grid resolutions up to $2^{\prime}$ (about $5 \%$ deviations between the different simulations), whereas the $0.5^{\prime}$ and the $1^{\prime}$ grids agree well for the first 4 major peaks. The main reason for the increasing deviations with time is presumably reflections from under-resolved coastal regions. We conclude that the application of a $1^{\prime}$ grid is adequate for the propagation. The finest ComMIT grid has a resolution of $15 \mathrm{~m}$, which corresponds to the finest local bathymetry available to us. Preferably also the grid dependence of the ComMIT part of the simulations should have been assessed. However, a proper grid-refinement test involving all resolutions levels and coupling between the grids is a time consuming task that is left for the future. Still, we emphasize that $15 \mathrm{~m}$ is a very fine resolution that should yield reliable inundation predictions.

In Fig. (9), the effect of dispersion for the Northeastward propagation is clear, showing that the LSW model over predicts the amplitude by almost a factor of two. The time history of the surface elevation outside Bridgetown, in a depth of $50 \mathrm{~m}$, is displayed in Fig. (10). In contrast to waves propagating eastward into the Atlantic Ocean the effect of dispersion is negligible in the waves reaching Barbados. This is partly due to the smaller depths, $\sim 3000 \mathrm{~m}$, along the path of propagation towards Barbados ( $\sim 3000 \mathrm{~m})$ as compared to up to more than $6000 \mathrm{~m}$ depths in the deep ocean. However, directionality and effects linked to the continental margin probably contribute as well. Furthermore, we see that the highest elevation, closer to $0.5 \mathrm{~m}$, appear roughly 84 minutes after the earthquake.

The maximum values of the surface elevation during the whole simulation are given in the left panel (Fig. 11). The values range from about $1.3 \mathrm{~m}$ to slightly less than $3 \mathrm{~m}$. When it comes to the effect of a tsunami striking populated areas, a critical parameter is the flow depth (the height of water above the ground). The maximum flow depth in Bridgetown for the Lesser Antilles scenario is found in the right panel in Fig. (11), ranging from 0 to about $2.5 \mathrm{~m}$. The highest flow depth is here found on the shoreline. It should be noted that buildings and infrastructure are not considered in the simulations. Such obstructions may locally increase the flow depths or channelize the water, hence intensifying the water current velocities and the wave loads. On the other hand, they may also increase the flow resistance. Here, we have assumed a high tide and a small amount of possible sea level rise $(0.35 \mathrm{~m})$ due to climate changes, in total $0.7 \mathrm{~m}$ [40].

\section{CONCLUDING REMARKS}

We have applied our combination of dispersive propagation model and the NLSW ComMIT model for coastal impact to two cases studies, involving slide generated and earthquake generated waves, respectively. In the first case dispersion was crucial for the evolution of the tsunami, including the trans-Atlantic propagation. In the latter case the propagation model allowed us to assess the effect of the dispersion that varied between ignorable and crucial, according to direction, propagation distance and depth. It was also shown that simulations including both dispersive long distant tsunami propagation as well as local runup is swift on a standard standalone computer. As a bonus we could model the earthquake source flexibly in the propagation model, without being restricted by the predefined, though often very useful, unit sources of ComMIT.

We believe that tsunami computation will be inceasingly dependent on concerted application of a variety of specialized models, dealing with source, tsunami generation, propagation, runup and interaction with structures during 


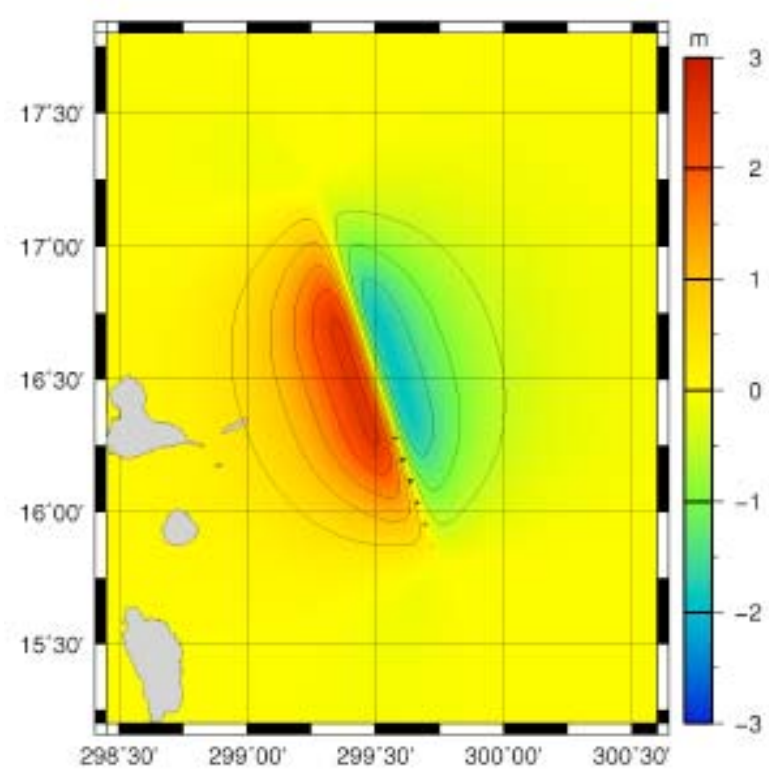

(a)

Surface elevation after 30 minutes

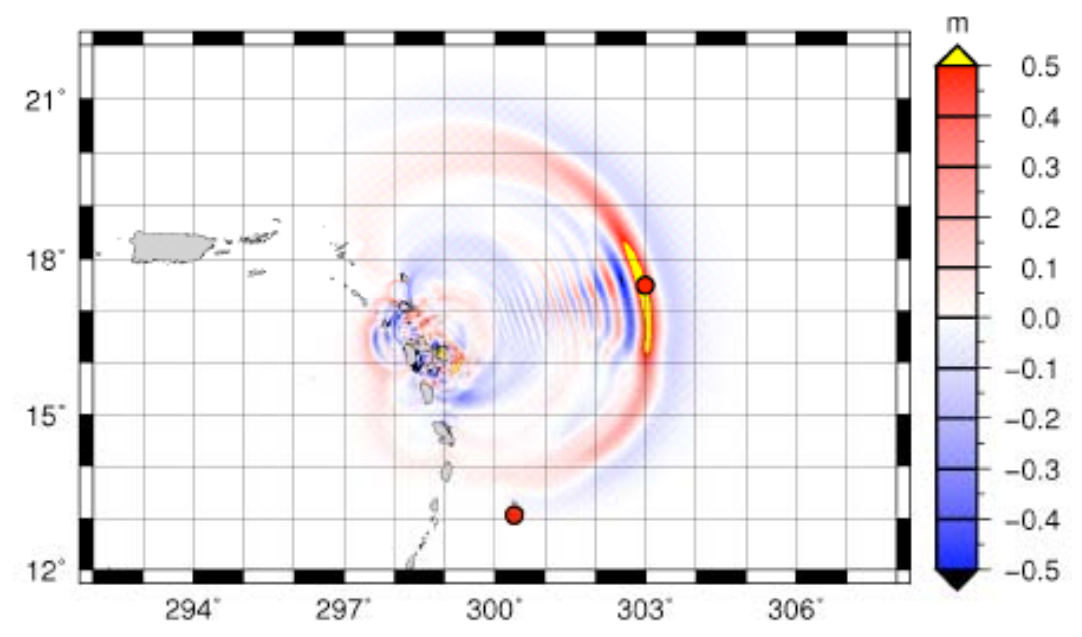

(b)

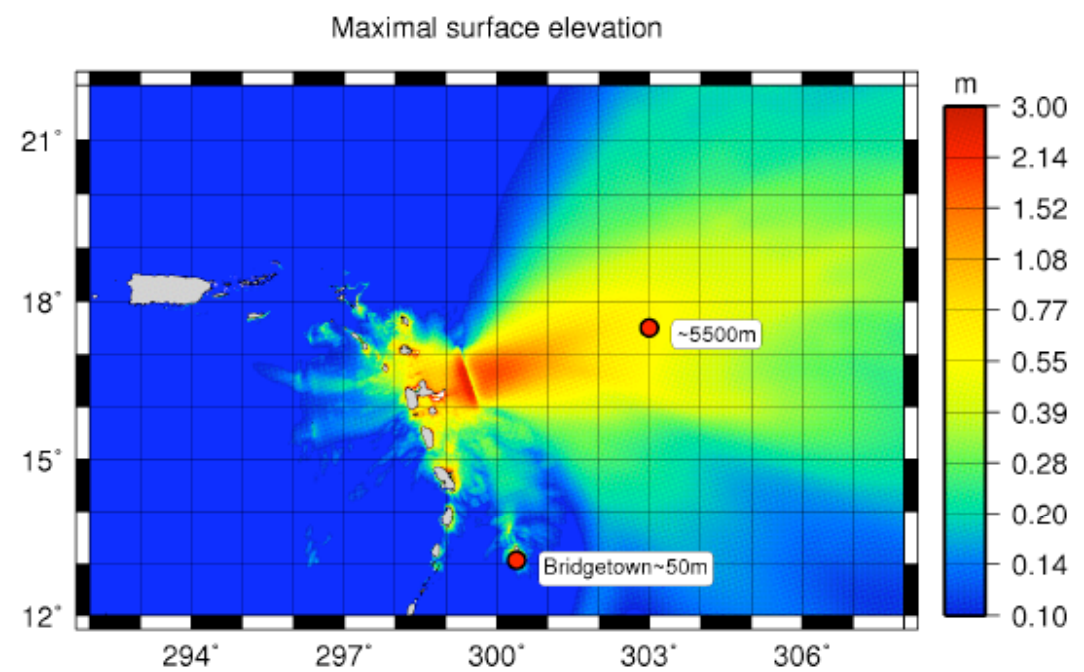

(c)

Fig. (7). Tsunami propagation for the lesser antilles scenario. Upper panel shows the intial condition. Mid panel shows a snapshot after 30 minutes of propagation. In the lower panel the corresponding maximum surface elevation during 6 hours of tsunami propagation is found. Note that the scale in the lower panel is logarithmic. 


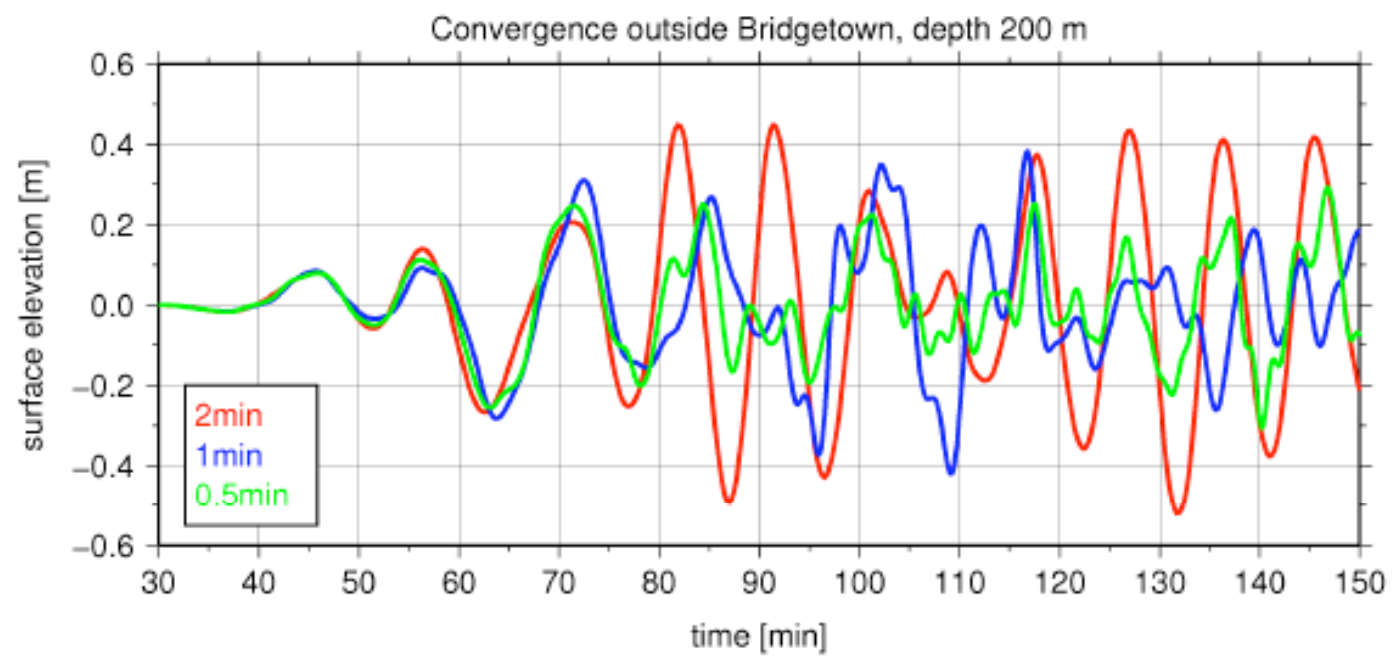

Fig. (8). Time series for the surface at $200 \mathrm{~m}$ depth outside Bridgetown. The legend refers to the spatial grid increment in minutes.

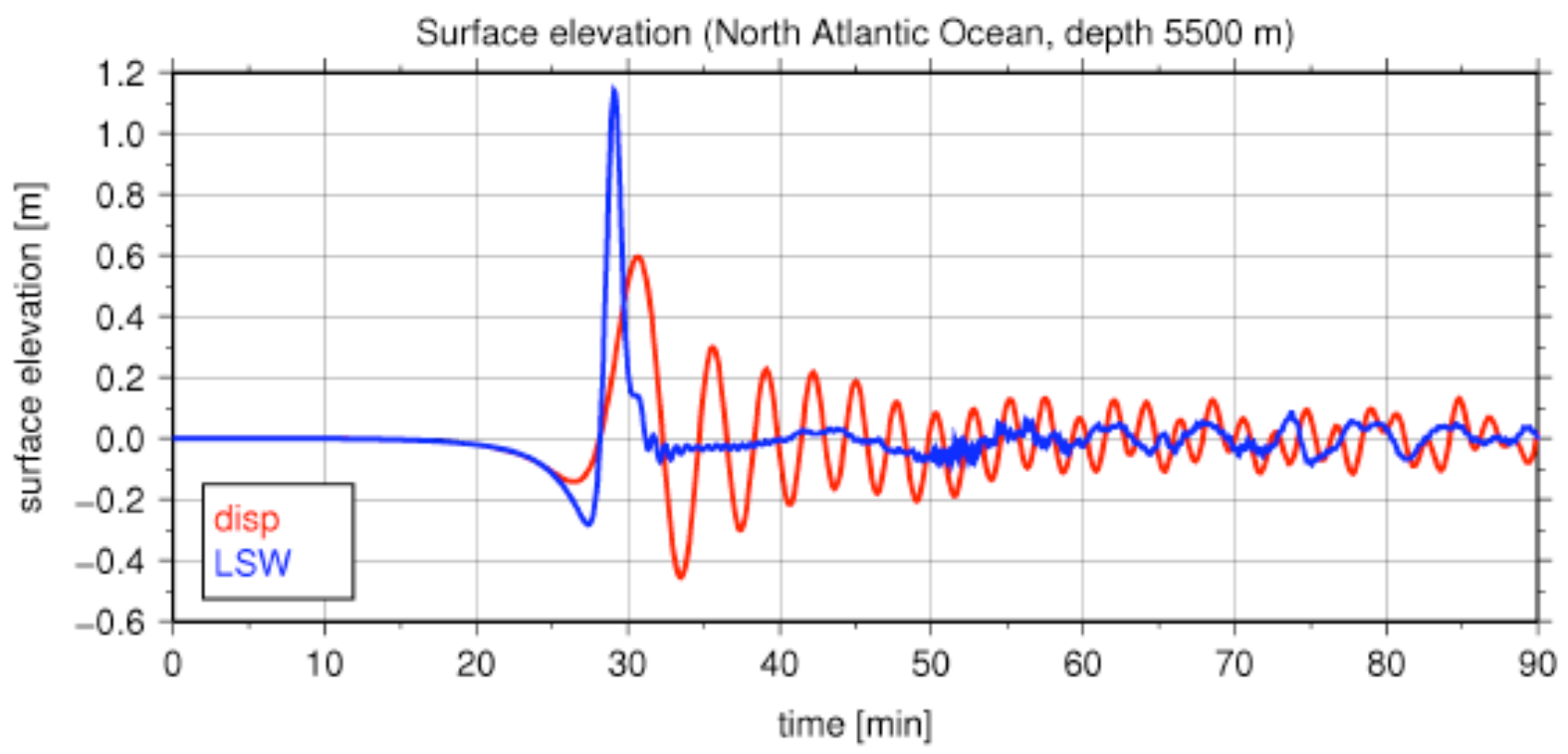

Fig. (9). The effect of dispersion for the lesser antilles scenario at the Northeast location, (see Fig. 7). "LSW"' and "disp" are the linear hydrostatic (linear shallow water) and linear dispersive descriptions, respectively.

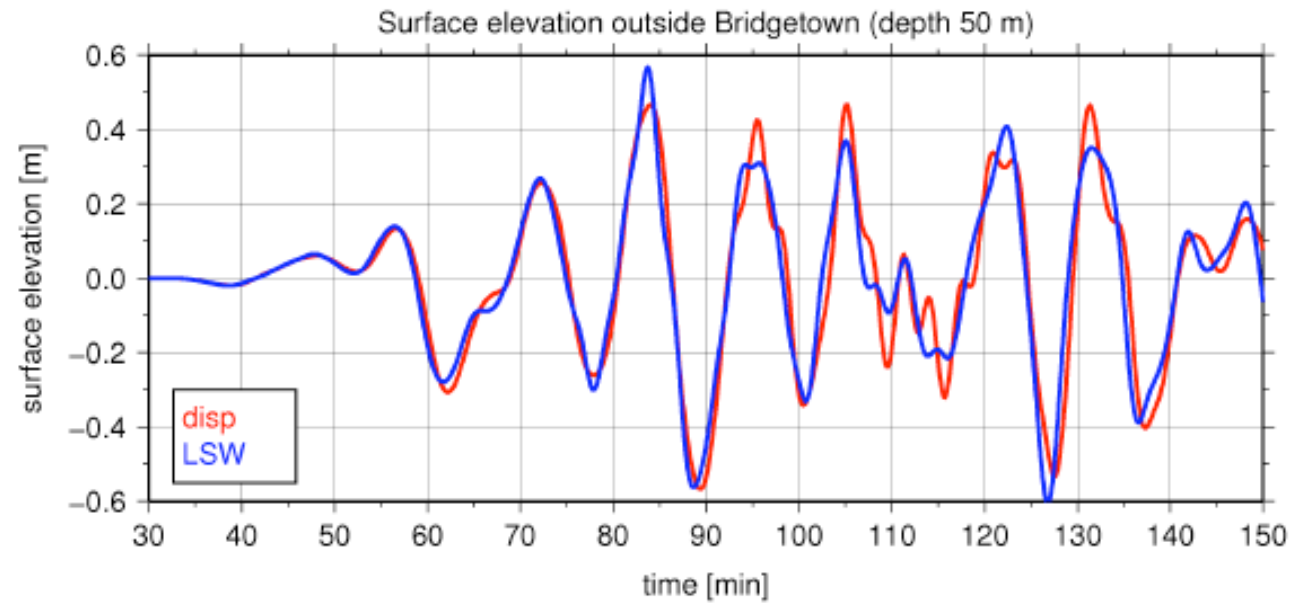

Fig. (10). Surface elevation calculated by the propagation model at a gauge outside Bridgetown. The sea depth is approximately 50 m. "LSW" and "disp" are the linear hydrostatic (linear shallow water) and linear dispersive descriptions, respectively 


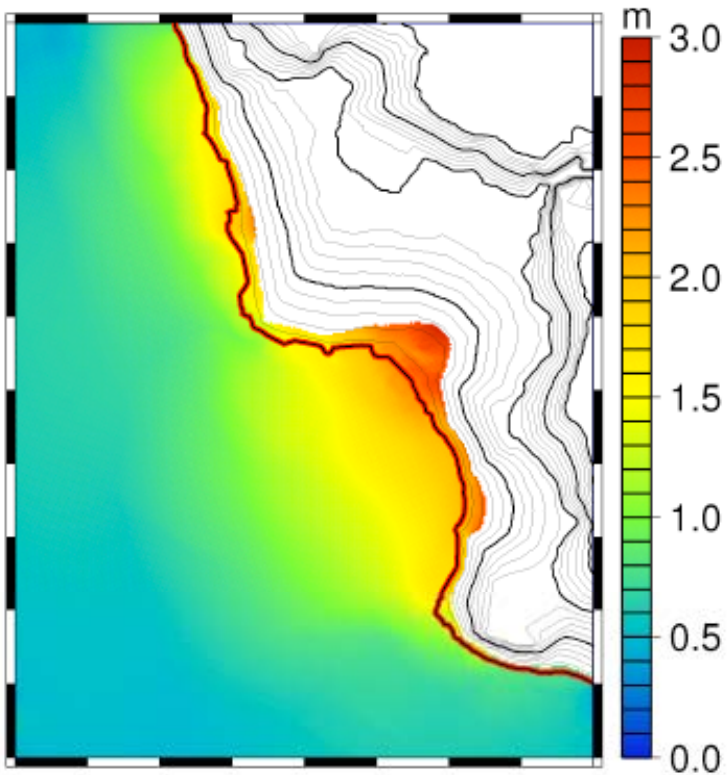

(a)

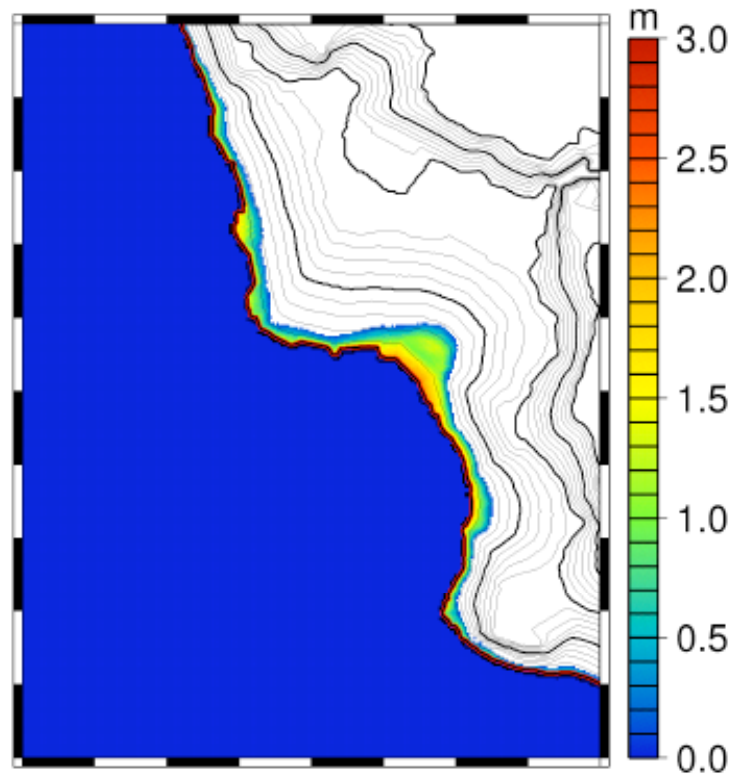

(b)

Fig. (11). Simulated inundation in bridgetown to the lesser antilles scenario. Left panel, maximal surface elevation during runup. Right panel, the calculated maximum flow-depth. Flow-depth seaside the shoreline is set to $0 \mathrm{~m}$.

inundation. To some extent such a combination may be undertaken manually, but in the long run a standarized, flexible and easy-to-use framework must be established. In our view the ComMIT system is a first push toward such a framework and is already an attractive tool with its present features.

\section{ACKNOWLEDGMENTS}

This work was financed by ICG (International Centre for Geohazards), as well as by NGI. This support was highly appreciated. The authors would like to thank Carl Harbitz for helpful comments to the manuscript. We also thank Vasily Titov and one anonymous reviewer for their comments leading to an improved manuscript. Finally, the authors are indebted to Chris Moore, Chris Chamberlin and Vasily Titov, for sharing the ComMIT and MOST models, and for the installation and help in using ComMIT. This paper is ICG publication number 269.

\section{REFERENCES}

[1] ComMIT. URL: http://nctr.pmel.noaa.gov/ComMIT/

[2] Titov VV, Synolakis CE. Modeling of breaking and non breakinglong-wave evolution and run up using VTCS-2. J Waterway Port Coast Ocean Eng 1995;121(6): 308-16.

[3] Titov VV, Synolakis CE. Numerical modeling of tidal wave runup. J Waterway Port Coast Ocean Eng. 1998; 124(4):157-71.

[4] COMCOT URL. Available from: http://ceeserver.cee.cornell.edu/ pll-group/comcot.htm

[5] Imamura F. In: Yeh H, Liu PLF, Synolakis C, Eds. Simulation of wave-packet propagation along sloping beach by TUNAMI-code. Long-wave runup models. World Scientific; 1996; pp. 231-41.

[6] LeVeque RJ, George DL. High resolution finite volume methods for the shallow water equations with bathymetry and dry states. In: Liu PLF, Yeh H, Synolakis C, Eds. Advanced numerical models for simulating tsunami waves and runup. Advances in Coastal and Ocean Engineering. World Scientific 2008; Vol. 10.

[7] Pedersen G. Modeling runup with depth integrated equation models. In: Liu PLF, Yeh H, Synolakis C, Eds. Advanced numerical models for simulating tsunami waves and runup. Advances in Coastal and Ocean Engineering. World Scientific 2008.
[8] Burwell D, Tolkova E, Chawla A. Diffusion and dispersion characteristics of a numerical tsunami model. Ocean Model 2007; 19(12): 10-30.

[9] Pedersen G, Løvholt F. Documentation of a global Boussinesq solver. Department of Mathematics, University of Oslo, Norway; 2008. Available from: http://www.math.uio.no/eprint/appl_math/ 2008/01-08.html

[10] Yoon SB. Propagation of distant tsunamis over slowly varying topography. J Geophys Res Oceans 2002; 10(10): 3140.

[11] Yoon SB, Lim CH, Choi J. Dispersion-Correction Finite Difference Model for Simulation of Transoceanic Tsunamis. Terr Atmos Ocean Sci 2007; 18(1): 31-53.

[12] Liu PLF, Yeh H, Synolakis CE. Advanced numerical models for simulating tsunami waves and runup. Advances in coastal and ocean engineering. World Scientific 2008; Vol. 10.

[13] Peregrine DH. Calculation of the development of an andular bore. J Fluid Mech. 1966; 22(2): 321-30.

[14] Peregrine DH. Long waves on a beach. J Fluid Mech. 1967; 77: 417-31.

[15] Nwogu O. Alternative form of Boussinesq equations for nearshore wave propagation. J Waterway Port Coast Ocean Eng. 1993; 119(6): 618-38.

[16] Wei G, Kirby JT, Grilli ST, Subramanya R. A fully nonlinear Boussinesq model for surface waves. Part 1. Highly nonlinear unsteady waves. J Fluid Mech. 1995; 294: 71-92.

[17] Kennedy AB, Chen Q, Kirby JT, Dalrymple RA. Boussinesq modeling of wave transformation, breaking, and run-up. Part I: 1D. J Waterway Port Coast Ocean Eng. 2000; 126(1): 39-47.

[18] Lynett PJ, Wu TR, Liu PLF. Modeling wave runup with depthintegrated equations. Coast Eng. 2002; 46: 89-107.

[19] Madsen PA, Schäffer HA. A review of Boussinesq-type equations for surface gravity waves. Advances in coastal and ocean engineering. Singapore: World Scientific 1999; Vol. 5: pp. 1-95.

[20] Kirby JT, Wei G, Chen Q, Kennedy AB, Dalrymple RA. Fully nonlinear Boussinesq wave model documentation and user's manual. Center for applied Coastal research, Department of Civil Engineering, University of Delaware, Newark, DE 19716; 1998. CACR-98-06. Available from: http://chinacat.coastal.udel.edu

[21] Kirby JT. FUNWAVE software download page; 1998. Available from: http://chinacat.coastal.udel.edu

[22] Shi F, Dalrymple RA, Kirby JT, Chen Q, Kennedy A. A fully nonlinear Boussinesq model in generalized curvilinear coordinates. Coast Eng 2001; 42: 337-58.

[23] Lynett PJ, Liu PLF. COULWAVE Model Page; 2004. Available from: http://ceprofs.tamu.edu/plynett/COULWAVE/default.htm 
[24] Kirby, J. T., F. Shi, P. Watts, and S. T. Grilli (2004), Propagation of short, dispersive tsunami waves in ocean basins, Eos Trans. AGU, 85(47), Fall Meet. Suppl., Abstract OS21-E02.

[25] Pedersen G. A Lagrangian model applied to runup problems. In: Liu PLF, Yeh H, Synolakis CE, Eds. Advanced numerical models for simulating tsunami waves and runup edited. Advances in Coastal and Ocean Engineering. World Scientific Publishing 2008; vol. 10.

[26] Lynett P, Liu PLF. A numerical study of the run-up generated by three-dimensional landslides. J Geophys Res 2005; 110: C03006.

[27] Pedersen G, Rygg OB. Numerical solution of the three dimensional Boussinesq equations for dispersive surface waves. Dept of Mathematics, University of Oslo; 1987.

[28] Rygg OB. Nonlinear refraction-diffraction of surface waves in intermediate and shallow water. Coast Eng 1988; 12: 191-211.

[29] Beji S, Nadaoka K. A formal derivation and numerical modelling of the improved Boussinesq equations for varying depth. Ocean Eng. 1996; 23(8): 691-704.

[30] Mesinger F, Arakawa A. Numerical methods used in atmospheric models. Publ Ser WMO 1976; 17:1-64.

[31] Løvholt F, Pedersen G. Instabilities of Boussinesq models in nonuniform depth. Int J Numer Methods Fluids 2009; 61(6): 606 - 37
[32] Pedersen G. A note on tsunami generation by earthquakes. Dept. of Mathematics, University of Oslo, Norway; 2001; no. 4.

[33] Pedersen G. Finite difference representations of nonlinear waves. J Numer Methods Fluids 1991; 13: 671-90.

[34] Masson DG, Harbitz CB, Wynn RB, Pedersen G, Løvholt F. Submarine landslides processes, triggers and hazard prediction. Phil Trans R Soc 2006; 364: 2009-39.

[35] Ward SN, Day S. Cumbre Vieja Volcano - Potential collapse and tsunami at La Palma, Canary Islands. Geophys Res Lett 2001; 28(17): 3397-400

[36] Mader CL. Modeling the La Palma Island tsunami. Sci Tsunami Hazards. 2001; 19(3):150-70.

[37] Gisler G, Weaver R, Gittings M. SAGE calculations of the tsunam threat from La Palma. Sci Tsunami Hazards 2006; 24: 288-301.

[38] Løvholt F, Pedersen G, Gisler G. Oceanic propagation of a potential tsunami from the La Palma Island. J Geophys Res Oceans 2008; 113 .

[39] NGI. Natural disaster mitigation in the Caribbean: Local tsunami risk assessment the Bridgetown - Demonstration project. Norwegian Geotechnical Institute, Report no. 20061575-3, 2009

[40] NGI. Natural disaster mitigation in the Caribbean: Regional tsunami exposure assessment. Norwegian Geotechnical Institute, Report no. 20061575-1, 2009.

Received: October 20, 2009

(C) Løvholt et al.; Licensee Bentham Open.

This is an open access article licensed under the terms of the Creative Commons Attribution Non-Commercial License (http://creativecommons.org/licenses/ by-nc/3.0/) which permits unrestricted, non-commercial use, distribution and reproduction in any medium, provided the work is properly cited. 